\title{
Accumulating brisk walking for fitness, cardiovascular risk, and psychological health
}

\author{
MARIE MURPHY, ALAN NEVILL, CHARLOTTE NEVILLE, STUART BIDDLE, and ADRIANNE HARDMAN \\ School of Applied Medical Sciences and Sports Studies, University of Ulster, Jordanstown, Northern Ireland, UNITED \\ KINGDOM; School of Sport, Performing Arts and Leisure, University of Wolverhampton, Walsall, UNITED KINGDOM; \\ and Department of Physical Education, Sports Science and Recreation Management, Loughborough University, \\ UNITED KINGDOM
}

\begin{abstract}
MURPHY, M., A. NEVILL, C. NEVILLE, S. BIDDLE, and A. HARDMAN. Accumulating brisk walking for fitness, cardiovascular risk, and psychological health. Med. Sci. Sports Exerc., Vol. 34, No. 9, pp. 1468-1474, 2002. Purpose: To compare the effects of different patterns of regular brisk walking on fitness, risk factors for cardiovascular disease, and psychological well-being in previously sedentary adults. Methods: Twenty-one subjects (14 women), aged $44.5 \pm 6.1 \mathrm{yr}$ (mean $\pm \mathrm{SD}$ ) were randomly assigned to two different, 6-wk programs of brisk walking in a cross-over design, with an interval of 2 wk. One program comprised one 30-min walk per day, $5 \mathrm{~d} \cdot \mathrm{wk}^{-1}$ (long bout) and the other three 10-min walks per day, also $5 \mathrm{~d} \cdot \mathrm{wk}^{-1}$ (short bouts). All walking was at $70-80 \%$ of predicted maximal heart rate. Maximal oxygen uptake $\left(\mathrm{V}_{2 \max }\right)$, body composition, resting arterial blood pressure, fasting plasma lipoprotein variables, and psychological parameters were assessed before and after each program. Results: Overall, subjects completed $88.2 \pm 1.1 \%$ and $91.3 \pm 4.1 \%$ of prescribed total walking time in the short- and long-bout programs, respectively. Both programs increased plasma concentrations of high-density lipoprotein cholesterol, and decreased concentrations of triacylglycerol and total cholesterol (all $P<0.05$ ). There were no changes in body mass, but the sum of four skinfolds, waist circumference, and hip circumference were decreased after both walking programs (all $P<0.05)$. Predicted $\dot{\mathrm{VO}}_{2 \text { max }}$ increased with both programs $(P<0.05)$, but this increase was greater with the program based on short bouts $(P<0.05)$. Both walking patterns resulted in similar decreases in tension/anxiety $(P<0.05)$. Conclusion: These findings suggest that three short bouts $(10 \mathrm{~min})$ of brisk walking accumulated throughout the day are at least as effective as one continuous bout of equal total duration in reducing cardiovascular risk and improving aspects of mood in previously sedentary individuals. Key Words: EXERCISE, INTERMITTENT, CONTINUOUS, AEROBIC FITNESS, CARDIOVASCULAR HEALTH, FATNESS, LIPIDS, MOOD
\end{abstract}

$\mathrm{D}$ espite the well-documented benefits of physical activity for physical and psychological health, the majority of the population of Northern Ireland remain inactive, with $80 \%$ of women and $70 \%$ of men not meeting current recommendations (18).

Adherence to structured exercise programs based on regular bouts of $30 \mathrm{~min}$ or more is poor (19), and so it has been suggested that people may be more likely to maintain a physically active lifestyle if they undertake several short bouts of exercise at intervals throughout the day (3). This suggestion is based on the principle, enshrined in current public health recommendations, that frequent short bouts of moderate-intensity physical activity confer health benefits commensurate with those derived from fewer but longer bouts of activity of equivalent total duration (22).

Walking is a popular and readily accessible form of moderate intensity physical activity, suitable for almost all the sedentary population. Epidemiological studies show that regular walking, independent of participation in more vig-

0195-9131/02/3409-1468/\$3.00/0

MEDICINE \& SCIENCE IN SPORTS \& EXERCISE E

Copyright (C) 2002 by the American College of Sports Medicine

Submitted for publication September 2001.

Accepted for publication April 2002.

DOI: 10.1249/01.MSS.0000027686.50344.77 orous exercise, is associated with a lower risk of coronary events (17) and type II diabetes (11). This study extends the literature in several important ways. Although some studies indicate that both short and long bouts of brisk walking can increase aerobic fitness among overweight or obese subjects $(5,12)$, there is little evidence in relation to other healthrelated outcomes such as blood lipids or among subjects of more normal weight. In addition, few studies comparing short and long bouts of exercise have used a multidisciplinary approach.

The purpose of the present study was therefore to test the hypothesis that short intermittent bouts of walking could elicit similar improvements in aerobic fitness, aspects of cardiovascular risk (body composition, plasma lipoproteins, and blood pressure), and indices of psychological health (self-efficacy and affect) of sedentary adults as one longer continuous bout. Previous studies in our laboratory suggested that the individual's perception of the pattern of activity to which they are assigned in a randomized trial may affect their motivation to adhere to the program. To overcome this potential bias, we employed a cross-over design so that each subject would be required to undertake both patterns of activity to provide a valid comparison of adherence. A 6-wk training period was selected as the minimum period over which alterations in fitness (10) and blood lipids (8) might be observed. 


\section{METHODS}

Design. The effects of two different 6-wk programs of brisk walking were compared, using a cross-over design. Each program involved subjects walking briskly at $70-80 \%$ of predicted maximal heart rate for a total of $30 \mathrm{~min} \cdot \mathrm{d}^{-1}$ on $5 \mathrm{~d} \cdot \mathrm{wk}^{-1}$. In one program, this was undertaken in a single, continuous session (long bout) and in the other in three, 10-min sessions separated by intervals of $\geq 3 \mathrm{~h}$ (short bouts). There was a wash-out period of 2 wk (range 14-17 d) between programs. The order in which the two programs were followed (short bouts/long bout or long bout/short bouts) was allocated randomly.

All measurements were made on four occasions, i.e., at baseline, after completion of the first walking program, before the second walking program, and on completion of the second program. Blood samples were obtained $2 \mathrm{~d}$ after completion of each training program.

Subjects. Thirty-two normolipidemic sedentary adults (20 women), aged $44.5 \pm 6.1 \mathrm{yr}$ (mean $\pm \mathrm{SD}$ ), were recruited through poster advertisement within the University of Ulster and the local community and a computer log-on message within the university. Sedentary was defined as not having engaged in more than $20 \mathrm{~min}$ of planned exercise per week during the preceding 3-month period. All subjects completed a confidential health-screening questionnaire. Exclusion criteria were a history of cardiovascular disease, type II diabetes mellitus, or of musculoskeletal problem preventing brisk walking; taking medication known to influence fat or carbohydrate metabolism; resting blood pressure $>150 \mathrm{~mm} \mathrm{Hg}$ systolic or $>105 \mathrm{~mm} \mathrm{Hg}$ diastolic; or a body mass index $(\mathrm{BMI})>35 \mathrm{~kg} \cdot \mathrm{m}^{-2}$. Ethical approval was obtained from the Research Ethical Committee of the University of Ulster, and written informed consent was obtained from all subjects, after a full explanation of the procedures and risks. During the study, nine subjects (5 men) withdrew. The reasons given were lack of time $(N=$ $4)$, loss of interest $(N=3)$, and injury or illness $(N=2)$. Three ( 3 men) withdrew during the first walking program (1 short-bouts/long-bout, 2 long-bout/short-bouts), two (1 man) during the wash-out period (1 short-bouts/long-bout, 1 long-bout/short-bouts), and four (1 man) (all long-bout/ short-bouts) during the second trial. Adequate compliance was defined as the completion of $60 \%$ of prescribed walks. This exceeds the usual compliance rates typically observed in structured exercise (4) and is consistent with other studies (2). Two women were excluded from the analysis because they failed to achieve adequate compliance. Data are therefore presented for 21 subjects (14 women).

Anthropometry and measurements of cardiovascular risk and psychological health. Standing height was measured using a stadiometer (SECA delta, Model 707, Birmingham, England) and body mass using an electronic balance $(200 \times 0.1 \mathrm{~kg}$, SECA, $)$. Waist and hip circumferences were measured using standard techniques (13). Skinfold thicknesses were measured using calipers at four sites (biceps, triceps, subscapular, and suprailiac) and summed as a measure of body fatness.
Resting arterial blood pressure was recorded as the mean of two measurements made using a random zero sphygmomanometer (Acuson MK2, Mountain View, CA), a minimum of 5 min apart, after the subject had been seated for at least 5 min.

Blood samples were obtained by venepuncture after a 12-h fast and with subjects in a semisupine position. Samples were separated, frozen at $-20^{\circ} \mathrm{C}$, and analyzed within 5 months for plasma total cholesterol, high-density lipoprotein (HDL) cholesterol, and triacylglycerol (TAG) concentrations using enzymatic colorimetric techniques (Boehringer Mannheim Ltd., East Sussex, U.K.). Accuracy and precision were maintained using quality control sera (Randox Ltd., Antrim, Northern Ireland). Within-batch coefficients of variation were $2.3 \%$ for TAG, $2.9 \%$ for $\mathrm{HDL}$ cholesterol, and $2.1 \%$ for total cholesterol. Blood pressure and blood lipid measurements were made by individuals blinded to subjects' group assignment.

Direct measurement of maximal oxygen uptake $\left(\mathrm{V}_{2 \max }\right)$ was considered ill-advised in sedentary subjects, some of whom exhibited a considerable burden of cardiovascular risk. A field walking test was therefore employed to monitor changes in aerobic fitness (21). Briefly, this involved subjects walking a distance of $2 \mathrm{~km}$ as quickly as possible in an indoor sports hall. Heart rate was monitored using shortrange telemetry (Sport-Tester, Polar Electro, Kempele, Finland). The time taken to complete the walk, mean heart rate during the last $30 \mathrm{~s}$ of the walk, age, and BMI were used to predict $\dot{\mathrm{V}}_{2 \text { max }}$, according to gender-specific equations (21).

Subjects completed three previously validated psychometric inventories. Mood was assessed using the abbreviated Profile of Mood States (28), which contained 27 items grouped into six subscales (tension/anxiety, depression, vigor, anger, confusion, and fatigue) (28). Responses were recorded on a 5-point Likert scale with scores of $0-4$ representing "not at all," "a little," "moderately," "quite a bit," and "extremely." The Barriers to Exercise scale (26) was based on a 5-point Likert scale with scores of 1-5 representing "strongly disagree" to "strongly agree." Subjects were required to indicate their agreement or disagreement to 15 suggested barriers to regular brisk walking, which were then grouped into four subscales (effort, time, health, and obstacles) (26). The magnitude of perceived self-efficacy was determined using Treasure and Newbery's (29) scale, modified to refer to walking rather than cycling. Subjects were required to indicate on a 10-point Likert scale (scores of 0-9 ranging from "not confident" to "very confident") their confidence in performing eight different types of physical activity (walking and nonwalking activities). The eight items were analyzed individually and then grouped into two subscales (walking activity and other physical activity) and reanalyzed.

Training programs. Training was self-governed and performed outdoors on the campus of the University of Ulster or near the subjects' homes. Subjects monitored their heart rate immediately after each bout of walking by palpation of the carotid or radial artery for $10 \mathrm{~s}$. Those who had 
TABLE 1. Physical characteristics of subjects at baseline (mean \pm SD)

\begin{tabular}{|c|c|c|}
\hline & $\begin{array}{c}\text { Short-Bouts/Long-Bout } \\
\text { Order }(N=13)\end{array}$ & $\begin{array}{c}\text { Long-Bout/Short-Bouts } \\
\text { Order }(N=8)\end{array}$ \\
\hline Men/women $(M)$ & $5 / 8$ & $2 / 6$ \\
\hline Age $(y r)$ & $43.8 \pm 6.6$ & $45.5 \pm 5.5$ \\
\hline Height (cm) & $166.0 \pm 7.8$ & $163.3 \pm 7.7$ \\
\hline Body mass $(\mathrm{kg})$ & $74.7 \pm 17.4$ & $71.6 \pm 11.7$ \\
\hline Body mass index $\left(\mathrm{kg} \cdot \mathrm{m}^{-2}\right)$ & $26.8 \pm 4.2$ & $26.7 \pm 2.7$ \\
\hline Sum of 4 skinfold thicknesses (mm) & $64.6 \pm 18.9$ & $62.1 \pm 12.4$ \\
\hline Waist circumference $(\mathrm{cm})$ & $88.1 \pm 14.3$ & $83.8 \pm 9.6$ \\
\hline Hip circumference (cm) & $104.2 \pm 10.2$ & $104.2 \pm 8.3$ \\
\hline Systolic blood pressure (mm Hg) & $128.5 \pm 14.3$ & $120.1 \pm 16.4$ \\
\hline Diastolic blood pressure $(\mathrm{mm} \mathrm{Hg})$ & $87.0 \pm 9.8$ & $78.9 \pm 10.1$ \\
\hline $\begin{array}{l}\text { Predicted } \dot{\mathrm{V}} \mathrm{O}_{2 \max } \\
\quad\left(\mathrm{mL} \cdot \mathrm{kg}^{-1} \cdot \mathrm{min}^{-1}\right)\end{array}$ & $26.5 \pm 5.5$ & $27.5 \pm 3.5$ \\
\hline Total cholesterol $\left(\mathrm{mmol} \cdot \mathrm{L}^{-1}\right)$ & $5.5 \pm 1.1$ & $5.31 \pm 0.6$ \\
\hline $\mathrm{HDL}$ cholesterol $\left(\mathrm{mmol} \cdot \mathrm{L}^{-1}\right)^{\prime}$ & $1.26 \pm 0.4$ & $1.31 \pm 0.5$ \\
\hline Triacylglycerol $\left(\mathrm{mmol} \cdot \mathrm{L}^{-1}\right)$ & $1.45 \pm 0.9$ & $1.52 \pm 0.7$ \\
\hline
\end{tabular}

Short-bouts pattern $=$ three 10 -min walks $\cdot d^{-1}$ on $5 d \cdot w^{-1}$; long-bout pattern $=$ one 30 -min walk $\cdot d^{-1}$ on $5 d \cdot w^{-1}$; no significant differences between groups at baseline.

difficulty used a short-range telemetry device (PE4000 Sport-Tester, Polar Electro). Subjects recorded the time of day, the duration of each walk, and the heart rate elicited in a training diary, which was submitted each week. Subjects undertook to refrain from planned exercise other than the walking programs and not to alter their diet.

Statistical analysis. Changes in the physical, physiological, and plasma lipoprotein data were assessed using a three-factor $(2 \times 2 \times 2)$ repeated-measures analysis of variance (ANOVA) with one between-individuals factor (program order) and two within-individual factors, i.e., time (before and after training) and pattern of walking (shortbouts vs long-bout).

For the psychological parameters, all items within a given subscale were checked for internal consistency using Cronbach's alpha. Alpha coefficients for all items in a subscale were deemed acceptable based on the standard criterion of 0.70 for such psychometric tests. Mean, standard deviation, and standard error were calculated for each subscale of each measure by using a statistical software package (Microsoft Excel 97, Microsoft Corporation). Friedman's test and Dunn's multiple comparison test were used to identify differences between the two patterns of walking and differences before and after training on mood, barriers to physical activity, and self-efficacy scores. The comparative statistical analyses were carried out using a statistical software package (Graphpad Prism version 2.01, Graphpad Software Inc., San Diego, CA). For all analyses, significance levels were set at $\mathrm{P}<0.05$. Results are presented as mean and standard deviation, unless otherwise stated.

\section{RESULTS}

Some characteristics of the study population at baseline are presented in Table 1. Random assignment of subjects to the two orders of presentation (long-bout/short-bouts or short-bouts/long-bout) resulted in two groups that did not differ in any measured characteristic. Overall, subjects completed $88.2 \pm 1.1 \%$ and $91.3 \pm 4.1 \%(P>0.05)$ of the prescribed total walking time in the short- and long-walk programs, respectively. Subjects were active on $28.3( \pm 1.6)$ and $27.5( \pm 1.5)$ days when undertaking the short- and long-walk programs, respectively. The group who undertook the short-bout/long-bout order completed an average of $139 \pm 3.0 \mathrm{~min} \cdot \mathrm{wk}^{-1}$ of brisk walking over the first $6 \mathrm{wk}$ and $136 \pm 6.3 \mathrm{~min} \cdot \mathrm{wk}^{-1}$ over the second $6 \mathrm{wk}$, i.e., $92.6 \pm$ $2.2 \%$ and $90.9 \pm 4.2 \%$ of the prescribed amount. The group who undertook the long-bout/short-bouts order completed an average of $128 \pm 8 \mathrm{~min} \cdot \mathrm{wk}^{-1}$ over the first $6 \mathrm{wk}$ and 129 $\pm 4 \mathrm{~min} \cdot \mathrm{wk}^{-1}$ over the second $6 \mathrm{wk}$, i.e., $85.1 \pm 5.7 \%$ and $86.2 \pm 3.1 \%$ of the prescribed amount. Although on average subjects assigned to the short/long order undertook more walking than those assigned to the long/short order, this difference was not statistically significant. Table 2 shows characteristics of subjects before and after the first and second walking programs, irrespective of the order in which short and long walks were undertaken.

Table 3 compares measurements made at baseline with those made after the different walking programs, for all subjects together. No significant changes in body mass were observed. The sum of four skinfolds, waist and hip circumference, and diastolic blood pressure all decreased significantly with both programs. The time taken to complete the 2-km walk decreased significantly after short-bouts (19.5 \pm 1.6 vs $17.7 \pm 1.6 \mathrm{~min})$ and long-bout programs $(18.5 \pm 1.8$ vs $18.1 \pm 1.8 \mathrm{~min}$ ) (both $P<0.05$ ). Predicted $\mathrm{VO}_{2 \max }$ increased significantly with both programs, but this increase was greater after the short-bouts pattern $(14.2 \%$ or $3.95 \pm$ $\left.3.21 \mathrm{~mL} \cdot \mathrm{kg}^{-1} \cdot \mathrm{min}^{-1}\right)$ than after the long-bout pattern $(3.8 \%$ or $1.1 \pm 3.21 \mathrm{~mL} \cdot \mathrm{kg}^{-1} \cdot \mathrm{min}^{-1}, P<0.05$ ) (Fig. 1). Subjects assigned to the long-bout/short-bouts order showed similar increases in $\mathrm{V}_{2 \text { max }}$ after each 6-wk program, whereas subjects assigned to the short-bouts/long-bout order showed a greater increase with the short-bout pattern than with the long-bout pattern $(P<0.05)$. This interaction is illustrated in Figure 2.

Fasting plasma concentrations of total cholesterol and TAG decreased significantly and HDL cholesterol concentration increased significantly with both programs, but the pattern of brisk walking did not influence the magnitude of these changes.

Changes in mood state, self-efficacy, perceived barriers, and postexercise feelings. Table 4 shows mean scores for the psychological parameters before after each walking program. Feelings of tension/anxiety decreased significantly after short-bout $(0.55 \pm 0.19$ vs $0.26 \pm$ $0.09, P<0.05)$ and long-bout programs $(0.48 \pm 0.13 \mathrm{vs}$ $0.20 \pm 0.07, P<0.05)$, with no difference in the magnitude of this change between programs. No other significant differences in mood state were evident with either walking program.

Before and after training, irrespective of pattern of walking, subjects reported greater confidence in walking than in other activities (cycling, jogging, and stair climbing).

The mean scores for the perceived barriers to physical activity of effort, time, obstacles, and health decreased numerically with both programs. However, these reductions were only statistically significant for the effort barrier (3.5 \pm 0.2 vs $2.5 \pm 0.2, P<0.05$ after short-bouts and long-bout 
TABLE 2. Predicted maximal oxygen uptake, anthropometric measures, blood pressure, and fasting plasma concentrations of total cholesterol, HDL cholesterol, and triacylglycerol of subjects before and after first and second walking program $(N=21$; mean \pm SD).

\begin{tabular}{|c|c|c|c|c|}
\hline & $\begin{array}{l}\text { Pre } 1^{\text {st }} \\
\text { Program }\end{array}$ & $\begin{array}{l}\text { Post } 1^{\text {st }} \\
\text { Program }\end{array}$ & $\begin{array}{l}\text { Pre } 2^{\text {nd }} \\
\text { Program }\end{array}$ & $\begin{array}{l}\text { Post 2nd } \\
\text { Program }\end{array}$ \\
\hline $\begin{array}{l}\text { Predicted VंOMDNM } \\
\quad\left(\mathrm{mL} \cdot \mathrm{kg}^{-1} \cdot \mathrm{min}^{-1}\right)\end{array}$ & $26.9(4.8)$ & $30.7 \quad(6.7)$ & $29.5(6.3)^{\star}$ & $30.8(6.0)$ \\
\hline Body mass $(\mathrm{kg})$ & 73.5 (15.2) & $73.6(15.7)$ & $73.4(16.0)$ & $73.6 \quad(16.1)$ \\
\hline Sum of 4 skinfold thicknesses (mm) & 63.7 (16.1) & $61.7(16.4)$ & $62.9(17.2)$ & $61.2(17.4)$ \\
\hline Waist circumference (mm) & $86.4(12.6)$ & $84.9(12.8)$ & $85.7(13.0)$ & $84.8(12.4)$ \\
\hline Hip circumference (mm) & $104.2(9.2)$ & $103.1(9.2)$ & $103.3(9.7)$ & $102.0(9.5)$ \\
\hline Systolic blood pressure (mm Hg) & $125.3(15.3)$ & $124.0(15.5)$ & $125.6(14.4)$ & $123.8(13.2)$ \\
\hline Diastolic blood pressure (mm Hg) & $84.0(10.4)$ & $83.3(9.1)$ & $84.0(7.7)$ & $81.7(7.6)$ \\
\hline Total cholesterol $\left(\mathrm{mmol} \cdot \mathrm{L}^{-1}\right)$ & $5.43(0.95)$ & $5.08(0.89)$ & $5.39(1.08)$ & $5.07(1.06)$ \\
\hline $\mathrm{HDL}$ cholesterol $\left(\mathrm{mmol} \cdot \mathrm{L}^{-1}\right)^{\prime}$ & $1.28(0.40)$ & $1.33(0.41)$ & $1.30(0.39)$ & $1.44(0.51)$ \\
\hline Triacylglycerol $\left(\mathrm{mmol} \cdot \mathrm{L}^{-1}\right)$ & $1.47(0.65)$ & $1.33(0.72)$ & $1.43(0.57)$ & $1.18(0.58)$ \\
\hline
\end{tabular}

* Significantly different than preintervention.

programs, respectively) and for the health barrier $(2.1 \pm 0.1$ vs $1.4 \pm 0.1, P<0.05)$.

\section{DISCUSSION}

This study complements previous findings that regular brisk walking improves aerobic fitness in sedentary people, whether undertaken in multiple short bouts or in fewer, longer bouts (20). The novel findings of the present study were that short bouts of brisk walking, taken at intervals throughout the day, also decreased aspects of cardiovascular risk and improved psychological well-being in a similar manner to a program based on 30-min bouts of walking. These findings are particularly pertinent to discussions on the efficacy of physical activity recommendations as they derive from study of middle-aged men and women drawn from a population with a high incidence of coronary heart disease (18), many of whom carried a measurable burden of cardiovascular risk.

Changes in plasma lipoprotein concentrations were similar with both patterns of walking and, although small (mean decreases in total cholesterol of 0.27 and $0.39 \mathrm{mmol} \cdot \mathrm{L}^{-1}$ and mean increases in HDL cholesterol of 0.07 and 0.12 $\mathrm{mmol} \cdot \mathrm{L}^{1}$ after short- and long-bout programs, respectively), may be expected to reduce the risk of coronary heart disease in both sexes (34). They were apparent after only $6 \mathrm{wk}$ of training, in line with earlier findings for HDL cholesterol. After an initial decline during the first $2 \mathrm{wk}$ of training at $70 \%$ of $\dot{\mathrm{VO}}_{2 \max }$, HDL concentrations have been reported to increase at a rate of approximately $0.03 \mathrm{mmol} \cdot \mathrm{L}^{-1} \cdot \mathrm{wk}^{-1}$ for $8 \mathrm{wk}$ (8). The decreases in plasma TAG concentration (means of 0.13 and $0.16 \mathrm{mmol} \cdot \mathrm{L}^{-1}$ after short- and longbout programs, respectively) we observed are consistent with the reciprocal relationship between TAG and HDL cholesterol. Findings of other studies of brisk walking (whether accumulated through short bouts or through longer sessions) and blood lipids vary, however. Whereas some have found changes $(5,14)$, others have not $(24,35)$. These inconsistencies may be attributed to differences such as preintervention lipid profiles (6) or perhaps the timing of blood sampling. In the present study, subjects refrained from exercise for $1 \mathrm{~d}$ before sampling. If alterations in blood lipids persist for up to $48 \mathrm{~h}$ after 45 min of walking at $60 \%$ $\mathrm{VO}_{2 \max }$ (16), our findings probably encompass some effect of recent exercise (9). However, the timing of the blood samples in our study was deliberately chosen. If people adopt a habit of walking on most days of the week (22), then the longest interval between brisk walks for exercise will be between 36 and $48 \mathrm{~h}$. Blood samples drawn in the morning after one day without brisk walking, as in the present study, reflect this scenario most closely.

Readiness to take up an exercise program may coincide with increased motivation to alter other health behaviors (19). Our subjects gave an undertaking not to modify their diet and reported at the conclusion of the study that they honored this undertaking. If, however, they did modify their diet, one might expect some substitution of dietary energy from fat with carbohydrate. This would decrease HDL cho-

TABLE 3. Anthropometric measures, blood pressure, predicted maximal oxygen uptake, and fasting plasma concentrations of total cholesterol, HDL cholesterol, and triacylglycerol of subjects before and after short-bouts and long-bout patterns $(N=21$; mean $\pm S D)$.

\begin{tabular}{|c|c|c|c|c|}
\hline & \multicolumn{2}{|c|}{ Short-Bouts Pattern $(3 \times 10 \mathrm{~min})$} & \multicolumn{2}{|c|}{ Long-Bout Pattern ( 1 × 30 min) } \\
\hline & Before & After & Before & After \\
\hline Body mass $(\mathrm{kg})$ & $73.4(15.5)$ & $73.7(16.0)$ & $73.7(15.8)$ & $73.5(15.7)$ \\
\hline Sum of 4 skinfold thicknesses (mm) & $62.3(17.2)$ & $61.3(17.0)^{*}$ & $63.7(16.4)$ & $61.6(17.1)^{\star}$ \\
\hline Waist circumference $(\mathrm{cm})$ & $86.1(12.7)$ & $85.8(13.0)^{\star}$ & $86.1(12.9)$ & $85.1(12.4)^{\star}$ \\
\hline Hip circumference $(\mathrm{cm})$ & $103.5(9.5)$ & $102.7(9.2)^{\star}$ & $103.0(9.6)$ & $102.4(9.6)^{\star}$ \\
\hline Systolic blood pressure (mm Hg) & $124.2(15.0)$ & $124.3(14.9)$ & $126.7(14.6)$ & $123.5(14.0)$ \\
\hline Diastolic blood pressure $(\mathrm{mm} \mathrm{Hg})$ & $84.3(9.5)$ & $82.9(9.0)^{\star}$ & $83.6(8.8)$ & $82.1(7.8)^{\star}$ \\
\hline Predicted $\mathrm{VO}_{2 \max }\left(\mathrm{mL} \cdot \mathrm{kg}^{-1} \cdot \mathrm{min}^{-1}\right)$ & $27.4(5.4)$ & $31.3(6.6)^{*} \dagger$ & $29.0(6.0)$ & $30.1(6.1)^{\star}$ \\
\hline Total cholesterol $\left(\mathrm{mmol} \cdot \mathrm{L}^{-1}\right)$ & $5.33(0.97)$ & $5.06(0.90)^{\star}$ & $5.48(1.06)$ & $5.09(1.06)^{*}$ \\
\hline $\mathrm{HDL}$ cholesterol $\left(\mathrm{mmol} \cdot \mathrm{L}^{-1}\right)^{\prime}$ & $1.29(0.39)$ & $1.36(0.49)^{\star}$ & $1.30(0.41)$ & $1.42(0.44)^{*}$ \\
\hline Triacylglycerol $\left(\mathrm{mmol} \cdot \mathrm{L}^{-1}\right)$ & $1.40(0.83)$ & $1.27(0.69)^{\star}$ & $1.39(0.61)$ & $1.24(0.63)^{*}$ \\
\hline
\end{tabular}

Short-bouts pattern $=$ three 10 -min walks $\cdot d^{-1}$ on $5 d \cdot$ wk ${ }^{-1}$; long-bout pattern $=$ one 30-min walk $\cdot d^{-1}$ on $5 d \cdot$ wk ${ }^{-1}$.

${ }^{\star}$ Values after training significantly different from values before training $(P<0.05)$.

$\dagger$ Increase in predicted $\mathrm{VO}_{2 \max }$ significantly greater with short bouts than with long bout $(P<0.05)$. 


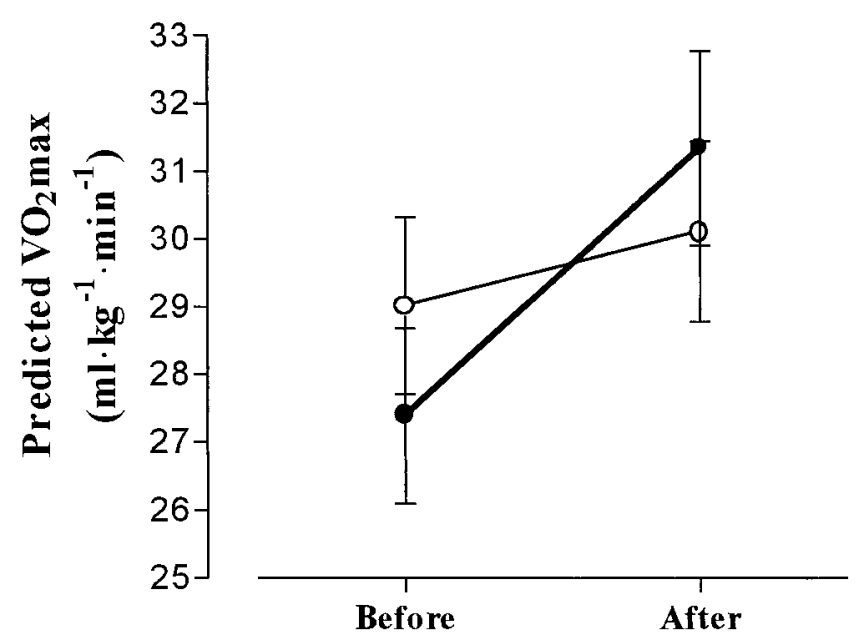

FIGURE 1-Predicted $\dot{\mathrm{V}} \mathrm{O}_{2 \max }$ before and after different patterns of walking $(N=21$; mean \pm SEM). Short-bouts (solid symbols, $\odot$ ), long-bout (open symbols, $\bigcirc$ ).

lesterol and increase TAG, i.e., the opposite of the changes we observed. Dietary change over the course of our study is thus an unlikely explanation for the changes in blood lipids.

Despite the theoretical net daily increase in energy expenditure associated with the walking programs (estimated at a total of $500-600 \mathrm{~kJ}$, depending on walking speed and

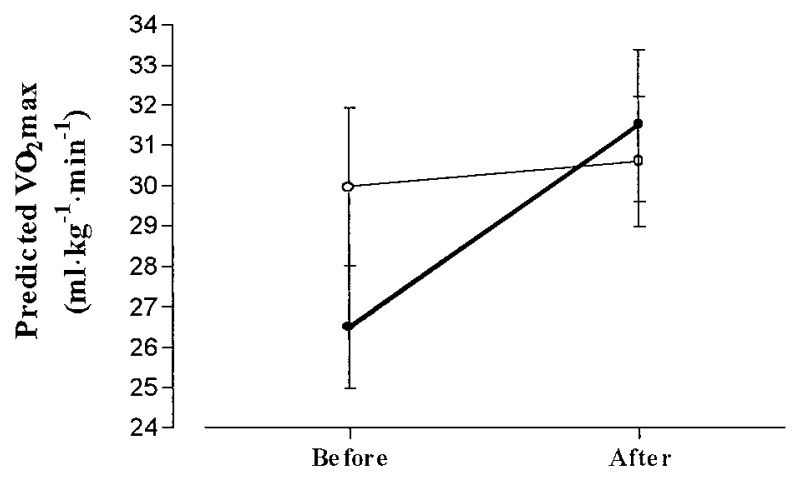

Short-bouts/long-bout order

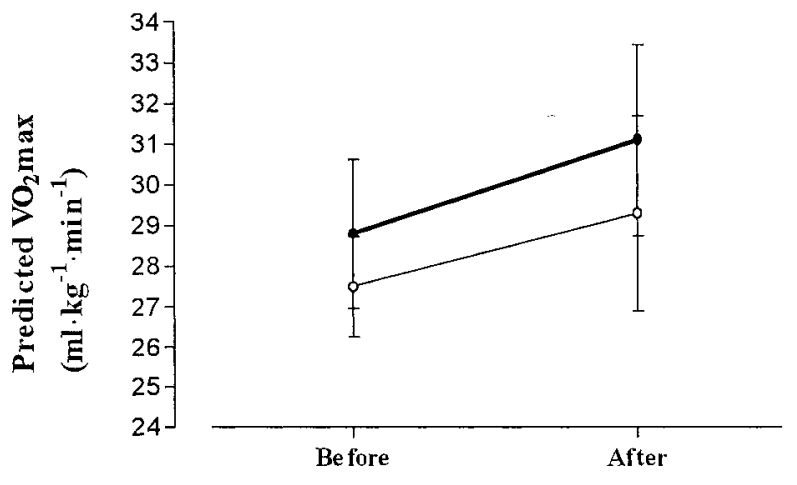

Long-bout/short-bouts order

FIGURE 2-Predicted $\dot{\mathrm{V}}_{2 \max }$ before and after short-bouts (solid

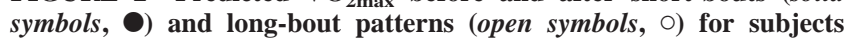
completing programs in short-bouts/long-bout order $(N=13)$ and long-bout/short-bouts order $(N=7)($ mean \pm SEM). body mass) (1), there was no decrease in body mass in this group of moderately overweight people. Nevertheless, the sum of skinfold thicknesses and waist and hip circumferences decreased significantly after both walking patterns. This is consistent with previous reports where decreases in body fat occurred in the absence of body mass changes (14) and may be indicative that brisk walking can alter body fat distribution. This, in its own right, may be expected to lower the risk of cardiovascular disease (15).

Both patterns of brisk walking lead to a decrease of about $1.5 \mathrm{~mm} \mathrm{Hg}$ in diastolic blood pressure. However, if this small reduction were achieved at a population level, it would have important public health implications for protecting against mortality from coronary heart disease and stroke (32).

An important finding was the significant reduction in feelings of tension/anxiety with brisk walking, irrespective of the pattern of this, and despite the fact that subjects were not particularly tense/anxious at baseline (mean score 0.62 \pm 0.20 (on a scale of $0-4$ )). Subjects with the highest tension/anxiety scores showed the greatest reduction with walking, and so, speculatively, this effect might be even stronger in individuals with greater levels of tension/anxiety. Although the stress-reducing effects of exercise are well documented (27), few studies have investigated brisk walking specifically. Ekkekakis et al. (7) found that one 10- to 15-min bout of walking was associated with shifts toward more pleasant affective states, with recovery periods showing greater calmness and less tension. The exact reasons for such shifts remain unclear but are likely to include enhanced feelings of confidence (23) as well as, speculatively, changes at the physiological $(23,30)$ and biochemical levels (25). We have been unable to find data on the effects of longer periods of regular walking for exercise.

Consistent with previous studies $(19,26)$, subjects reported effort and time as being two of the main barriers to physical activity. After participation in the walking program based on short bouts, the mean score for the effort barrier was markedly reduced. This was not observed after the long-bout program, which may imply that subjects found it less of an effort to maintain the routine of several short bouts of walking than of longer bouts. Indeed, subjects assigned to the short-bouts program first completed more walking and displayed less attrition in both patterns than the subjects assigned to the long-bout program first. Four subjects dropped out of the long-bout/short-bouts order during the second 6-wk period, whereas all of the subjects assigned to the short-bouts/long-bout order completed the second period of training. Speculatively, sedentary individuals may find it easier to maintain a program of 30 min bouts of brisk walking if first accustomed to several short bouts spread throughout the day. Moreover, the adherence and attrition patterns in the current study suggest that, after becoming accustomed to longer continuous bouts of brisk walking, subjects may find a more intermittent pattern less fulfilling. Future studies should attempt to consider whether undertaking a short bout walking program alters adherence to a subsequent program based on longer walks. From a public 
TABLE 4. Mood state, self-efficacy, and perceived barriers of subjects before and after short-bouts and long-bout patterns ( $N=21$; mean \pm SD).

\begin{tabular}{|c|c|c|c|c|}
\hline & \multicolumn{2}{|c|}{ Short-Bouts Pattern $(3 \times 10 \mathrm{~min})$} & \multicolumn{2}{|c|}{ Long-Bout Pattern $(1 \times 30 \mathrm{~min})$} \\
\hline & Before & After & Before & After \\
\hline \multicolumn{5}{|l|}{ Mood } \\
\hline Tension/anxiety & $0.55(0.19)$ & $0.26(0.09)^{*}$ & $0.48(0.13)$ & $0.20(0.07)^{*}$ \\
\hline Depression & $0.33(0.15)$ & $0.39(0.17)$ & $0.35(0.13)$ & $0.19(0.07)$ \\
\hline Anger & $0.26(0.16)$ & $0.12(0.08)$ & $0.22(12.9)$ & $0.09(0.05)$ \\
\hline Confusion & $0.31(0.15)$ & $0.10(0.05)$ & $0.24(0.10)$ & $0.19(0.09)$ \\
\hline Fatigue & $0.70(0.18)$ & $0.69(0.22)$ & $0.78(0.14)$ & $0.58(0.13)$ \\
\hline Vigor & $1.7(0.20)^{\prime}$ & $1.89(0.20)$ & $1.89(0.17)$ & $1.86(0.16)$ \\
\hline \multicolumn{5}{|l|}{ Self-efficacy } \\
\hline Walking & $5.8(0.8)$ & $6.6(0.6)$ & $5.8(1.0)$ & $6.7(0.7)$ \\
\hline Other activities & $3.8(0.8)$ & $4.7(0.9)^{\star}$ & $3.8(0.9)$ & $4.8(0.9)$ \\
\hline \multicolumn{5}{|c|}{ Perceived barriers to exercise } \\
\hline Effort & $3.5(0.2) \dagger$ & $2.5(0.2)^{*}$ & $2.7(0.2)$ & $2.2(0.1)$ \\
\hline Time & $3.0(0.3)$ & $2.9(0.2)$ & $3.2(0.2)$ & $2.6(0.2)$ \\
\hline Obstacles & $2.4(0.1)$ & $2.2(0.2)$ & $2.1(0.2)$ & $2.1(0.2)$ \\
\hline Health & $1.5(0.2) \dagger$ & $1.6(0.2)$ & $2.1(0.1)$ & $1.4(0.1)^{\star}$ \\
\hline
\end{tabular}

Short-bouts pattern $=$ three 10 -min walks $\cdot d^{-1}$ on $5 d \cdot$ wk ${ }^{-1}$; long-bout pattern $=$ one 30 -min walk $\cdot d^{-1}$ on $5 d \cdot$ wk ${ }^{-1}$.

* Values after training significantly different from values before training $(P<0.05)$.

$\dagger$ Values significantly different between short and long walks $(P<0.05)$.

health perspective, prescribing short bouts of walking for sedentary individuals may provide a useful stepping stone toward more traditional approaches to exercise.

This study augments the existing body of literature demonstrating that brisk walking improves aerobic fitness. Both walking programs were effective in decreasing the time taken to complete the $2-\mathrm{km}$ walk ( $-2: 48 \mathrm{~min}$ :s after shortbouts, $-0: 24 \mathrm{~s}$ after long-bouts). This accords well with Whitehurst and Menendez (33), who demonstrated that $8 \mathrm{wk}$ of brisk walking (20-40 min. $\left.\mathrm{d}^{-1}, 3 \mathrm{~d} \cdot \mathrm{wk}^{-1}\right)$ resulted in a 1:24-min:s decrease in the mean time taken to complete a 1-mile walk among older women. However, although predicted $\dot{\mathrm{V}}_{2 \max }$ increased after both walking programs in the present study, this increase was greater following the shortbouts pattern. This finding is contrary to that of Jakicic et al. (12), who reported similar increases in aerobic fitness after long and short bouts of exercise.

In the present study, $\dot{\mathrm{V}} \mathrm{O}_{2 \max }$ increased more with shortbout walks than with long (Fig. 1). This finding was confined to the group who followed the short-bout program first (Fig. 2) and may be explained by two factors. First, the tendency for subjects assigned to the short walks first completed more of the prescribed walking in both program may have increased the magnitude of improvement. A second explanation may relate to the study design, as Table 2 shows, $\dot{\mathrm{V}} \mathrm{O}_{2 \max }$ was significantly higher before the second intervention period than at baseline, most likely because the wash-out interval was too short for this particular outcome. It is well-established that increases in $\dot{\mathrm{VO}}_{2 \max }$ are greatest early in a training period, so the potential for improvement might have been less during the second period than during the first. This factor might explain why the effect of walking pattern on the increase in $\mathrm{V}_{2 \max }$ appeared to be influenced by the order in which the programs were followed: in the group who followed the short-bout program first, it would constrain the increase in $\dot{\mathrm{V}}_{2 \max }$ with long walks; in the group who followed the long-bout program first, it would constrain the increase in $\dot{\mathrm{VO}}_{2 \max }$ with short walks. Thus, the cross-over design, which worked well for other out- comes, is a limitation in the interpretation of our findings on $\dot{\mathrm{VO}}_{2 \max }$.

High attrition rates limit the potential public health benefits from physical activity. These often exceed 50\% during the first 3-6 months of structured exercise regimens (31). The drop-out rate in our study was lower than this $-28 \%$ over 3 months (combining both programs and neglecting the wash-out period), but still not trivial. The demands of the study may have inflated this above that which might be expected of people attempting to fulfil population guidelines for physical activity, although none of the drop-outs mentioned this as a reason. Among those who completed the study, adherence was high and did not differ systematically with pattern of walking; an average of nearly $90 \%$ of the prescribed walking time was completed in both programs. Consequently, our study provides suggestive evidence that encouraging brisk walking, regardless of pattern, may be an effective way to improve compliance with physical activity recommendations. The effects of these two patterns of brisk walking on longer-term maintenance have yet to be fully elucidated.

This multidisciplinary study provides further confirmation that three bouts of 10-min brisk walking accumulated over the course of the day are at least as effective in increasing aerobic fitness, improving blood lipid profiles, and enhancing aspects of psychological well-being of sedentary middle-aged adults as one longer bout of the same total duration.

The authors thank Mr. John Brown from the University of Ulster for assistance in the Human Performance Laboratory and Dr. Mairead O'Hare from the Institute of Clinical Science at the Queens University of Belfast for plasma lipid analyses. They also thank Jillian Davis and Kathy Ann Stevenson for help with data collection and fieldwork, and the subjects for their interest and participation.

Address for correspondence: Dr. Marie H. Murphy, School of Applied Medical Sciences and Sports Studies, University of Ulster (Jordanstown), Shore Road, Newtownabbey, Co. Antrim, Northern Ireland, BT37 0QB, United Kingdom; E-mail: mh.murphy@ ulst.ac.uk. 


\section{REFERENCES}

1. Ainsworth, B., W. L. Haskell, A. S. Leon, et al. Compendium of physical activities: classification of energy cost of human physical activities. Med. Sci. Sports Exerc. 25:71-80, 1993.

2. Biddle, S. J. H., and N. Mutrie. Psychology of physical activity: determinants, well-being and interventions. London: Routledge $1991 \mathrm{p} 44$.

3. Blair, S. N., H. W. Kohl, N. F. Gordon, and R. S. Paffenbarger. How much physical activity is good for health? Ann. Rev. Pub. Health. 13:99-126, 1992.

4. Dishman, R. K. Overview. In: Exercise Adherence: Its Impact on Public Health, R. K. Dishman (Ed.). Champaign, IL: Human Kinetics, 1988, pp. 1-9.

5. Donnelly, J. E., D. J. Jacobsen, K. Snyder Heelan, R. Seip, and S. Sмiтн. The effects of 18 months of intermittent versus continuous exercise on aerobic capacity, body weight and composition, and metabolic fitness in previously sedentary, moderately obese females. Int. J. Obes. 24:566-572, 2000.

6. Durstine, J. L., and W. L. Haskell. Effects of exercise training on plasma lipids and lipoproteins. In: Exercise and Sports Sciences Reviews, J. Holloszy (Ed.). Baltimore: Williams \& Wilkins, 1994, pp. 477-521.

7. Ekkekakis, P., H. E. Hall, L. M. Van Landuyt, and S. J. PetruZZELLO. Walking in (affective) circles: can short walks enhance affect? J. Behav. Med. 23:245-275, 2000.

8. FARrell, P. A., and J. BARBORIAK. The time course of alterations in plasma lipid and lipoprotein concentrations during eight weeks of endurance training. Atherosclerosis 37:231-238, 1980.

9. Haskell, W. L. Health consequences of physical activity: understanding and challenges regarding dose-response. Med. Sci. Sports Exerc. 26:649-660, 1994.

10. Hickson, R. C, J. M. Hagberg, A. A. Eshani, et al. Time course of adaptive responses of aerobic power and heart rate to training. Med. Sci. Sports Exerc. 13:17-20, 1981.

11. Hu, F. B., R. J. Sigal, J. W. Rich-Edwards, et al. Walking compared with vigorous physical activity and risk of type 2 diabetes in women: a prospective study. JAMA 282:1433-1439, 1999.

12. JAKICIC, J. M., C. Winters, W. LANG, and R. R. Wing. Effects of intermittent exercise and use of home exercise equipment on adherence, weight loss, and fitness in overweight women: a randomized trial. JAMA 282:1554-1560, 1999.

13. Jones, P. R. M., M. J. Hunt, T. P. Brown, and N. G. Norgan. Waist-hip circumference ratio and its relation to age and overweight in British men. Hum Nutr. Clin. Nutr 40:239-247, 1986.

14. KukKonen-Harjula, K., R. LaukKanen, et al. Effects of walking training on health-related fitness in healthy middle-aged adults -a randomised controlled study. Scand. J. Med. Sci. Sports 8:236242, 1998.

15. Lapidus, L., C. Bengtsson, B. Larsson, K. Pennert, E. Rybo, and L. SJöstrom. Distribution of adipose tissue risk of cardiovascular disease and death: a twelve year follow-up of participants in the population study of women in Gothenburg, Sweden. Br. Med. J. 289:1261-1263, 1984.

16. Lee, R., D. Nieman, R. Raval, J. Blankenship, and J. Lee. The effects of acute moderate exercise on serum lipids and lipoproteins in mildly obese women. Int. J. Sports Med. 12:537-542, 1991.

17. Lee, I. M., K. M. Rexrode, N. R. Cook, J. E. Manson, and J. E. BURING. Physical activity and coronary heart disease in women: is “no pain, no gain” passé? JAMA 285:1447-1454, 2001.
18. Macauley, D., E. E. Mccrum, G. Stott, et al. Northern Ireland Health and Activity Survey Report. Belfast: Her Majesty's Stationery Office, 1994.

19. Marcus, B. H., T. K. King, M. M. Clark, B. M. Pinto, and B. C. Bock. Theories and techniques for promoting physical activity behaviours. Sports Med. 22:321-331, 1996.

20. Murphy, M. H., and A. E. Hardman. Training effects of short and long bouts of brisk walking in sedentary women. Med. Sci. Sports Exerc. 30:152-157, 1998.

21. Oja, P., M. LaukKanen, M. Pasanen, T. Tyry, and I. Vuori. A 2-km walking test for assessing the cardiorespiratory fitness of healthy adults. Int. J. Sports Med. 12:356-362, 1991.

22. Pate, R. R., M. Pratt, and S. N. Blair. Physical activity and public health: a recommendation from the Centres for Disease Control and Prevention and the American College of Sports Medicine. JAMA 272:241-246, 1995.

23. RAGLIN, J. S. Exercise and mental health: beneficial and detrimental effects. Sports Med. 9:323-329, 1990.

24. Snyder, K. A., J. E. Donnelly, D. J. Jacobsen, G. Hertner, and J. M. JAKICIC. The effects of long-term, moderate intensity, intermittent exercise on aerobic capacity, body composition, blood lipids, insulin and glucose in overweight females. Int. J. Obes. 21:1180-1189, 1997.

25. Steinberg, H., E. A. Sykes, and N. Leboutiller. Exercise addiction: indirect measures of endorphins? In: Exercise Addiction: Motivation for Participation in Sport and Exercise, J. Annett, B. Cripps, and H. Steinberg (Eds.). Leeds: British Psychological Society, 1997, pp. 6-14.

26. Steinhardt, M. A., and R. K. Dishman. Reliability and validity of expected outcome and barriers for habitual physical activity. $J$. Occup. Med. 31:536-546, 1989.

27. Taylor, A. H. Physical activity, anxiety, and stress. In: Physical Activity and Psychological Well-Being, S. J. H. Biddle, K. R. Fox and S. H. Butcher (Eds.). London: Routledge, 2000, pp. 10-45.

28. Terry, P. C., A. M. Lane, H. J. Lane, and L. Keohane. Development and validation of a mood measure for adolescents. J. Sports Sci. 17:861-872, 1999.

29. Treasure, D. C., and D. M. Newbery. Relationship between self-efficacy, exercise intensity, and feeling states in a sedentary population during and following an acute bout of exercise. J. Sport Exerc. Psychol. 20:1-11, 1998.

30. Van Doornen, L. J., E. J. De Geus, and J. F. Orlebeke. Aerobic fitness and the physiological stress response: a critical evaluation. Soc. Sci. Med. 26:303-307, 1988.

31. Weinberg, R. S., and D. Gould. Foundations of Sport and Exercise: Psychology, 2nd Ed. Champaign, IL: Human Kinetics, 1999, p. 372 .

32. Whelton, P. K. Epidemiology of hypertension. Lancet 344:101106, 1994

33. Whitehurst, M., and R. N. Menendez. Endurance training in older women: lipid and lipoprotein responses. Physician Sportsmed. 19:95-103, 1991.

34. Wilson, P. W. F. High-density lipoprotein, low-density lipoprotein and coronary heart disease. Am. J. Cardiol. 66:7A-10A, 1990.

35. Woolf-May, K., E. M. Kearney, D. W. Jones, R. C. R. Davison, D. Coleman, and S. R. BIRD. The effect of two different 18-week walking programs on aerobic fitness, selected blood lipids and factor XIIa. J. Sports Sci. 16:701-710, 1998. 